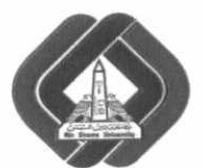

International Journal of Intelligent Computing and Information Science

\title{
FACIAL EXPRESSION RECOGNITION BASED ON PRINCIPAL COMPONENTS ANALYSIS
}

\author{
A. Hewa \\ O. Nomir \\ A. Saleh \\ Department of Computer Science, Faculty of Computer and Information, Mansoura University ,Egypt \\ Aramhewa8@gmail.com \\ omnomir@yahoo.com \\ elfetouh@gmail.com
}

\begin{abstract}
Recognizing facial expression is one of the most effective applications of image processing and has obtained great attention in latest years. A recognition system for facial expression is a computer based application which detects an individual facial expression for the purposes of authentication, criminal identification, passport verification, estimating age, and various other purposes. In this study, we propose a human recognition system based on facial expression. The system depends on extracting features using Principal Component Analysis (PCA) which later used in the training and recognition steps. The system is able to recognize diverse facial expressions such as Neutral, Anger, Disgust, Fear, Happy, Sad and Surprise. The primary objective of this study is to improve the efficiency and to achieve better recognition rate using Support Vector Machine (SVM). The system is evaluated using the registered JAFFE Dataset of face images. The results show that or proposed system is robust and maintain high recognition rate.
\end{abstract}

Keywords: Facial Expression, PCA, Feature Extraction, SVM, Recognition Rate, JAFFE Face Dataset

\section{Introduction:}

A recognition system based on facial expression is a computer application that detects the facial expressions of the various persons for the purposes of authentication, criminal identification, passport verification, age estimation, and various other purposes. A facial expression recognition system is a graphical user interface that can identify individuals based on their facial properties, thus recognizing the identity of a target. The research of facial expression recognition has great impractical value include subject of pattern recognition, a digital image processing (DIP), a computer vision, machine learning (ML), and physiology. It also has a high correlation with other biometrics recognition methods.

Principle Component Analysis (PCA) is a methodology of classic features extraction and data representation technique which is strongly uses in the pattern recognition applications. The purpose of the PCA is to reduce the large dimensionality data space into the minimal dimensionality features space to describe the data in the economical way [1]. Face is a multidimensional structure and needs strong computing techniques for recognition. Face recognition is an integral part of the biometrics [2]. The facial expression recognition can be performed by using the Principle Component Analysis (PCA) to extract facial features. PCA is a strong methodology which widely uses for dimensionally reduction in data space. First, PCA is applied to extract facial features for all images in a given database. Given a target image to get classified, PCA is applied to extract its facial features. After that, automatic classification is achieved to determine facial expression for a given image, neutral, anger, disgust, fear, happy, sad, or surprise (as shown in Figure(1)). This procedure for facial expressions classification will 
be able to classify a given image inside the used database or outside this database by using Support Vector Machine (SVM).

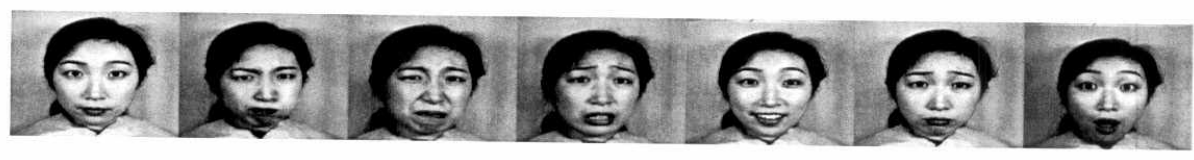

Figure 1. Examples of facial expressions from JAFFE Face Database images: Neutral, Anger, Disgust, Fear , Happy, Sad and Surprise (from left to right).

Nevertheless, the facial expression recognition research has attracted many researchers to this field and several successful research studies have been achieved in the past years. In [3],They proposed a system for facial expression recognition. They used a novel graph-preserving sparse nonnegative matrix factorization algorithm in their proposed system. They used the projected gradient method to calculate the nonnegative solution of the used algorithm. They applied the proposed system on the Cohn-Kanade database and the JAFFE database. The results of their system show that the novel graph-preserving sparse nonnegative matrix factorization algorithm provides best facial representations and achieves higher recognition rates than nonnegative matrix factorization.

In [4],They presented a super-resolution method that uses nonlinear mappings to infer coherent features that favor higher recognition of the nearest neighbor classifiers for recognition of single low resolution face image. The proposed method can compute super-resolved cohesive features conformable to an input low resolution image. That is according to the trained radial basis function model efficiently and accurately. They used for face recognition Super Resolution method in the feature domain, and they applied canonical correlation analysis to obtain the coherent subspaces between the holistic features of higher recognition and low resolution face images.

In [5], They proposed a tensor perceptual color framework for facial expression recognition. It based on information contained in color facial images. The proposed framework enables multi linear image analysis in different color spaces, and demonstrates that color components provide more information for robust facial expression recognition. The experimental results of their research demonstrate that color information has significant potential to improve emotion recognition performance due to the complementary characteristics of image textures.

In [6],They discussed a facial expression recognition method by evaluating eigen faces. A detailed mathematical formulation and entities used in principal component analysis are described. Most pertinent eigen face, eigen values and mean values are the entities used by principal component analysis method for matching.

In [7], They introduced a compression for many techniques for automated recognition of human face action. They compared the principal component analysis algorithm with singular value decomposition algorithm. The aim of this research is to show that principal component analysis is superior compared to singular value decomposition in terms of recognition rate. They used JAFEE and real database to evaluate the performance, and for recognized many principal expression such as angry, happy, sad, disgust and surprise.

In [8], They proposed a system for human face expression recognition based on improving current surveillance systems to make it detect a person's expression (who has intentions of causing harm) and report to the securities before the person can commit any for bidden work. The system catches ones 
feeling/expressions through a camera and send the footage for preprocessing before it can be extracted to look if the emotion/expression captured has the intentions of causing harm.

In [9], They proposed a method for human face expression recognition based on principal component analysis and singular value decomposition. They focused on solving these already existing recognition problems by recognizing the facial expression. Their research showed that the above dimensionality reduction algorithms have an encouraging recognition performance of recognizing images under different principal expressions and economically reducing data dimension thereby solving the problem of large database.

In [10], They investigated the performance of different feature extraction methods for human face expression recognition. These techniques are based on two approaches which are higher order local autocorrelation and local binary pattern. The results of their research showed that local binary pattern operator coefficients have surprisingly high information content.In this paper, we propose a human facial expression recognition system based on Principal Components Analysis (PCA), and Support Vector Machine(SVM).First each facial image in the given database is preprocessed, convert the RGB image to gray image and them resize the image.The PCA is applied to calculate the mean, deviation, covariance matrix, eigen vectors, eigen values and projection for each image in the database, followed by features extraction. Finally, the SVM is applied to classify the expression of the human face. At the beginning the given database is splitted into two sets, the first is used training, and the second is used for testing.

\section{Facial Expression Recognition System:}

This research introduces a facial expression recognition system to classify an individual's facial expression in a given image. The proposed system depends on three main processes: preprocessing, PCA and features extraction, and SVM classification as shown in Figure (2). The following three subsections describe in details the steps of the proposed system.

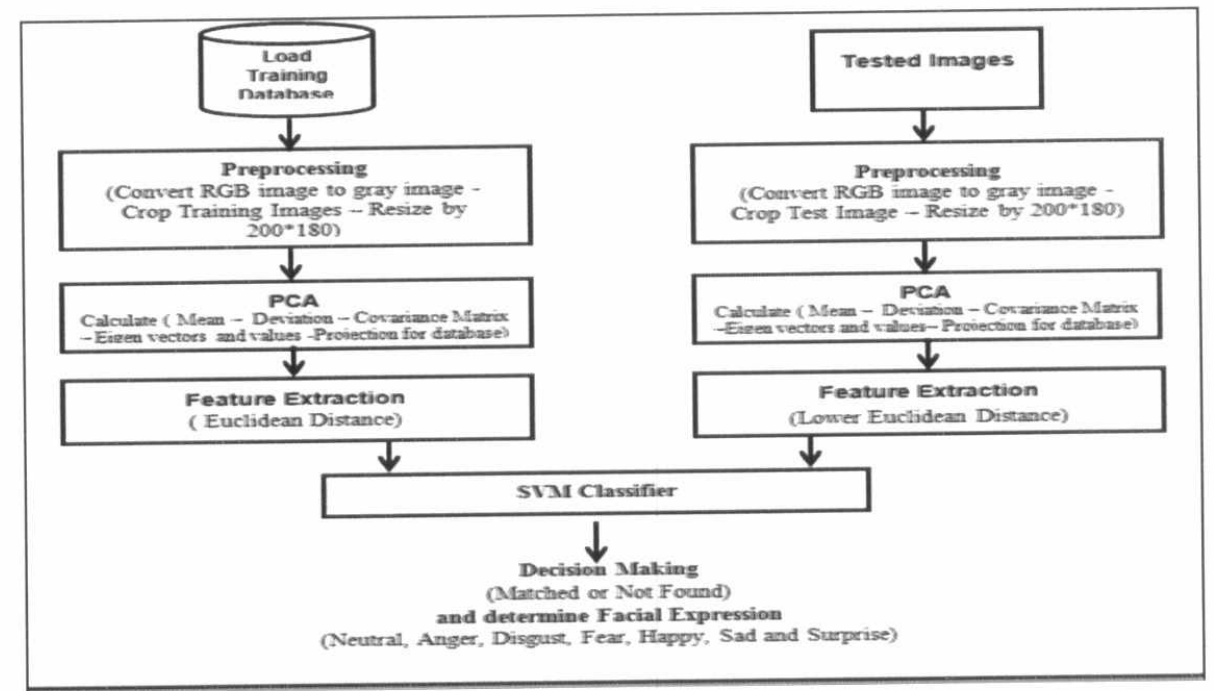

Figure 2. An overview of the proposed system 


\section{Pre-processing}

In this research, the pre-processing stage can be achieved in three steps. First all the RGB images in the database are converted to gray-scale images (these will be applied to the training dataset images as well as to the test dataset images). Then, all the images are automatically cropped. The image cropping step is shown in Figure (3). And finally, all the database images are resized to $200 * 180$ pixels, to prepare the image for the next stage, extracting the facial features.

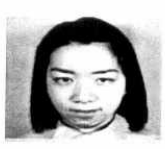

(a)

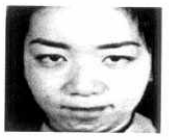

(b)

Figure 3. An example of image cropping. (a) The image before Cropping. (b) The image after cropping.

\section{The PCA and the Features Extraction}

Feature extraction in image processing mainly depends on reducing the amount of resources required to describe a large set of data. In this research paper, features are extracted using Principle Component Analysis (PCA).

\section{(Principal Component Analysis) PCA}

Principal Component Analysis (PCA) is a methodology that widely used in pattern recognition field and signal processing for data reduction and features extraction. Principal Components Analysis (PCA) is a way of identifying patterns in data, and expressing the data in such a way as to highlight their similarities and differences. Since patterns in data can be hard to find in data of high dimension, where the luxury of graphical representation is not available, PCA is a powerful tool for analyzing data. The main purpose of applying the PCA is to lower the dimensionality in a feature space.

A face image of size $\mathrm{N}^{*} \mathrm{~N}$ pixels can be considered as a one-dimensional vector of dimension $\mathrm{N}^{2}$. The primary idea of the principal component analysis is to find the vectors that better account for the allocation of image faces within the whole image space. Because these vectors are the eigenvectors of the covariance matrix corresponding to the original face images, and because they are face-like in appearance, they are referred to as "eigen faces"[11].

The following steps show the steps of applying the PCA algorithm for extracting facial features. 1.Prapare the training faces: Load $\mathrm{M}$ face images from the face database. Some sample face images are shown as Figure(4).
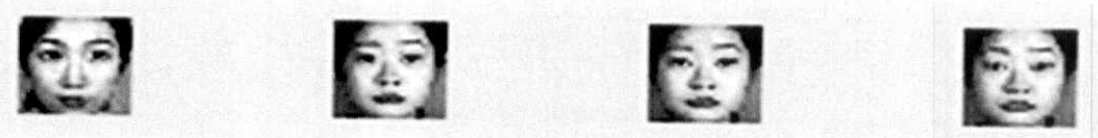

Figure 4. Samples from face database images

2. Compute the average face vector:Let the $M$ face images named as $X_{1}, X_{2}, \ldots, X_{M}$. The average (mean) for images face of the training images is defined by 


$$
\text { Mean_Image }=\frac{1}{M} \sum_{i=1}^{M} \mathrm{X}_{\mathrm{i}}, i=1,2, \ldots \ldots . M
$$

3. Calculate the covariance matrix: the average face image "mean image" is subtracted from the original face $\left(\mathrm{X}_{\mathrm{i}}\right)$, and the result is stored in the matrix $\left(\operatorname{dev}_{\mathrm{i}}\right)$.

$$
\operatorname{dev}_{i}=\mathrm{X}_{\mathrm{i}}-\text { Mean_Image }
$$

\begin{tabular}{|c|c|c|c|c|c|c|c|c|c|c|c|c|c|c|}
\hline 'ys' & 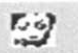 & 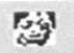 & 55i & EY & 焉: & {$[:]$} & (1) & {$\left[\begin{array}{l}0 \\
-1\end{array}\right.$} & "ي & 8 & 等: & 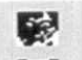 & 5 & \\
\hline 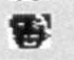 & 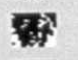 & 젔 & $i_{g}=7$ & 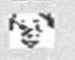 & ? & 접 & 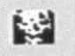 & (2) & (स) & 50 & ( ) & హ건 & 섯 & \\
\hline 3 & 웡 & : & 5 & 58 & 咸 & $\because 3$ & $F=$ & $\Leftrightarrow 1$ & 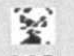 & (8) & 实 & 8 & 题 & \\
\hline & EI & 5 & 1 & {$\left[\begin{array}{l}3 \\
{[}\end{array}\right.$} & 'Ag & 핳 & {$[\mathrm{F}+\mathrm{i}$} & [럴 & 窟 & 㳇 & 商 & 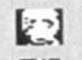 & & \\
\hline 2 & 장 & 8 & K8 & 崡 & 露 & 察 & $\Leftrightarrow 1$ & 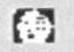 & 等。 & 裂. & 蛋 & & & \\
\hline $5 !$ & 8 & 63 & 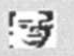 & '河 & [5] & 何 & 㹂] & [5] & (5) & 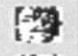 & 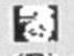 & $\mathbf{E}$ & 1 & \\
\hline 8 & 8 & 8 & 8 & 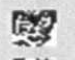 & 酸 & 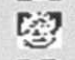 & $E$ & 6*1 & (5) & 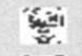 & 急. & $<$ & 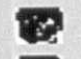 & \\
\hline 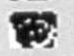 & rys. & dea & tys & 蛋 & [B] & [ & 8 & 8 & 89 & E) & 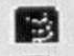 & 93 & 3 & \\
\hline 4 & 3 & 88 & T & 㥀 & E⿺辶力木 & 5 & (6) & [. 3 & (3) & [3] & 宽 & 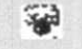 & 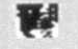 & \\
\hline$A$ & 陚 & (5) & 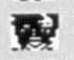 & 재앟 & 8 & sy & 然? & $I_{\frac{\bar{v}}{y}}$ & (지릴 & (3) & 1엉 & 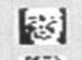 & {$\left[\begin{array}{l}{[-1} \\
{[-1}\end{array}\right.$} & \\
\hline 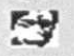 & 13 & Fु & 8 & 然 & 菣 & (1) & F) & $5 \geqslant$ & {$[D]$} & יب⿱亠乂 & 努" & 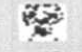 & 3 & \\
\hline 줄 & ? & 5 & 8 & (6) & 恣 & 象 & 得 & [战 & 핵 & 67 & 䎕 & 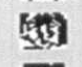 & $=1$ & \\
\hline 87 & 8 & F्व & 政 & $8 ?$ & 뎔 & {$[\because]$} & 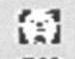 & {$[13]$} & 深 & 窎. & 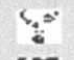 & (2) & 8 & \\
\hline-7 & 7 & 준? & [as? & 鹰? & , & 6 & 춨 & Eivid & (3) & 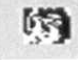 & 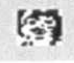 & E.) & $E d$ & \\
\hline
\end{tabular}

The result of subtracting each image in dataset with mean face images is shown in Figure (5).

Figure 5. Result subtract each image with mean face images

4. Find the covariance matrix for the image.

$$
\operatorname{cov}=\operatorname{dev} * \operatorname{dev}{ }^{\prime} ;
$$

5. Calculate the eigen values and eigenvectors: for a given image the function eig is applied on the calculated covariance matrix.

$$
\left[E_{-} \text {V,E_D }\right]=e i g(\operatorname{cov}) \text {; }
$$

where cov is the covariance matrix, E_V is the eigenvector matrix, and E_D is the eigen value matrix.

6. Sort the two matrices: sort the columns of the eigenvector matrix E_V, and the eigen value matrix E_D by descending eigen value.

Feature extraction converts pixel data into a higher-level representation of shape, color, texture, and spatial configuration of the face or its components.

The eigen vectors calculated in the steps $(5,6)$ will appear as light and dark areas inside image in a specific pattern. This pattern is how different features of a face image are extracted. Features extracted 
will evaluate matching facial expression between images, if there is any style of facial hair, where the hairline is, or evaluate the size of the nose, eyes or mouth. We use features extraction by using 28 eigen Faces as shown in figure (6) by searching in the eigen vector maximum value above threshold and this threshold more than $0.5 e+008$.

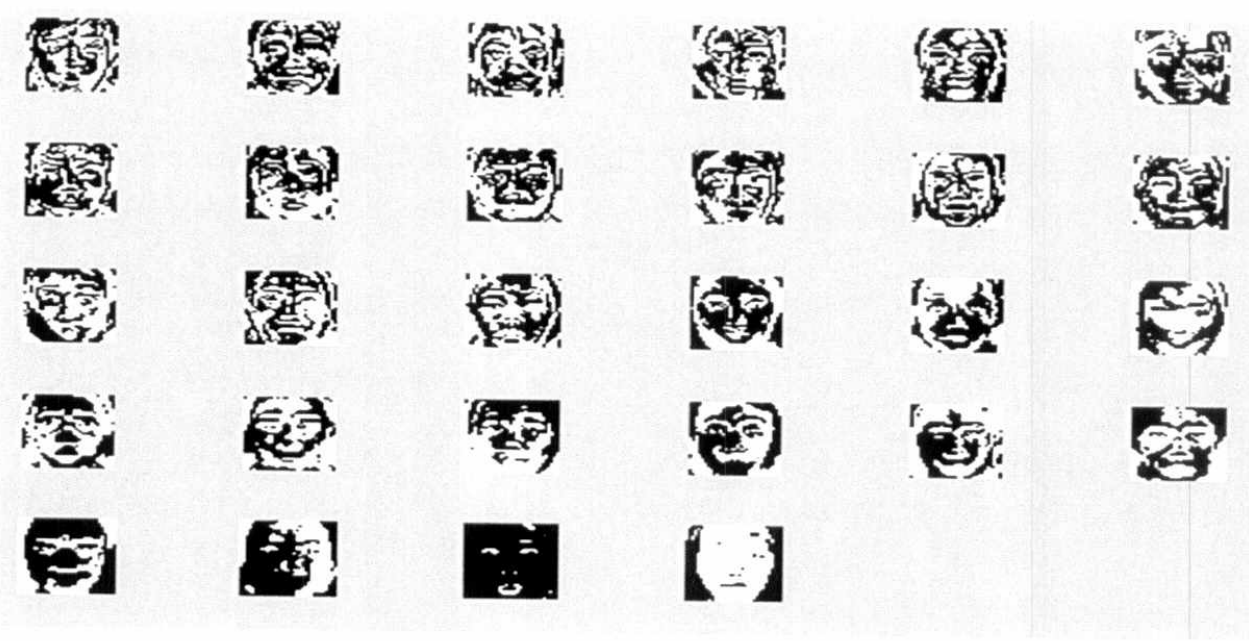

Figure 6. the eigen Faces $>$ that are greater than a pre-calculated threshold

\section{Classification Using (Support Vector Machine) SVM}

Support Vector Machine (SVM) classifier, is a popular method of classification [12].SVM was essentially designed for binary classification and here, in this research work, a multi-class classification is used.

SVM is a powerful tool and widely used in many areas, however it can only handle binary classification. Great effort has been put into finding how to effectively expand SVM for multi-class classification, and several techniques have been proposed [13] [14] [15] [16] [17].

The simplest idea may be to divide an n-class problem into a series of binary classifications. There are two ways: we can consider it either as n "one-against-all" classifications or as $C_{n}^{2}$ "one-against-one" problems. There are some other methods that consider all classes at once [16] [17]. Comparison in [13] has shown one-against-one is more suitable, because our problem is to classify a given image into one out of seven classes, so this is the method we used in this research work.

There are seven groups that training stage can handle which represent the seven possible facial expressions (Neutral, Anger, Disgust, Fear, Happy, Sad, and Surprise). The lower Euclidean distance is used to determine the closet input test image to anyone in the database.

We set groups matrix as $210 * 7$ with binary value 0,1 .

Neutral sets in the first column[ones(30,1);zeros(180,0)].

Anger sets in the second column [zeros(30,1);ones(30,1);zeros(150,1)]. 
Disgust sets in the third column [zeros $(60,1)$; ones $(30,1)$,zeros $(120,1)]$. Then we set another expressions like before expressions.

$\begin{array}{llllllll}\text { Groups }= & 1 & 0 & 0 & 0 & 0 & 0 & 0 \\ 0 & 1 & 0 & 0 & 0 & 0 & 0 \\ 0 & 0 & 1 & 0 & 0 & 0 & 0 \\ 0 & 0 & 0 & 1 & 0 & 0 & 0 \\ 0 & 0 & 0 & 0 & 1 & 0 & 0 \\ 0 & 0 & 0 & 0 & 0 & 1 & 0 \\ 0 & 0 & 0 & 0 & 0 & 0 & 1\end{array}$

Then we train between Features for database images, which we extracted with every group will represent every expressions by using SVM - training as this step:

model 1 =svmtrain(Features Dataset, Groups $(:, 1))$;

model_2 =svmtrain(Features_Dataset, Groups $(:, 2)) ; \ldots .$.

And do it for every expression

Then we test the features extraction from tested image with training model as this step

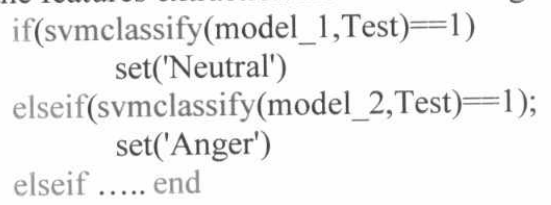

Then, the Euclidean distance is applied to find the face image with the lower Euclidean distance, which is given by.

$$
\text { Euclidean_Distance }=\sqrt{\sum_{\mathrm{i}=1}^{\mathrm{n}}\left(\mathrm{feat}_{-} \mathrm{X}_{\mathrm{i}}-\text { feat_test }_{\mathrm{i}}\right)^{2}}
$$

The feature vector of the input test image is feat test, and that of the feature for database images is feat_X, where $\mathrm{n}$ is the total number of features. A match is found by lowest euclidean_distance value to get the image having the facial expression closer to the tested image as shown as Figure(7).by calculating a covariance matrix by $x^{*} y$ in size, we get $x^{*} y$ eigen faces.

The image with the lowest Euclidean_distance value in facial expressions images will be represented as the eventual expression image(Neutral, Anger, Disgust, Fear, Happy, Sad and Surprise).

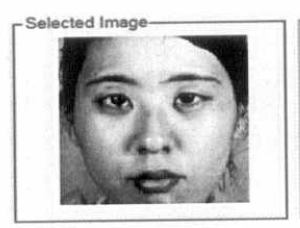

(a)

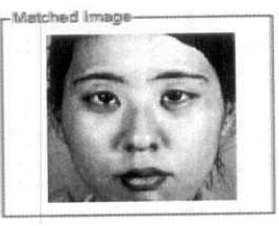

(b)

Figure 7. The matched image with the lower Euclidean distance. (a) The input image. (b) The matched image. 


\section{Result and Discussion}

Our proposed system was evaluated using the public JAFFE Face dataset images. The training database includes 140 face images, classified as 20 face images of neutral expression group, 20 face images of anger expression group, 20 face images of disgust expression group, 20 face images of fear expression group, 20 face images of happy expression group, 20 face images of sad expression group and 20 face images of surprise expression group. There are 70 testing images from JAFFE dataset,10 images for each of the seven facial expression.

The system was implemented under a platform MATLAB 2014,OS: windows 7, and CPU:intel $®$ Core TM i5-4200@1.60GHz.In this paper, we have providing an approach to crop a face only from whole image and resize it by using the same size is $200 \times 180$ for all training and testing face images. Then, the features are extracted by using PCA method with Support Vector Machine (SVM) as a classifier, and we have achieved the best results. And we have got time elapsed for (training, calculate Eigen value, calculate Euclidean distance and classification by using SVM Classifier) as shown as Table (1).

Table 1. Elapsed time for some steps in the proposed system in seconds

\begin{tabular}{|c|c|}
\hline Step & Elapsed Time(sec) \\
\hline $\begin{array}{c}\text { Load the database (140 face images) after } \\
\text { cropping and resizing to } 200^{*} 180\end{array}$ & 37.953 \\
\hline Calculate Eigen value & 1.2565 \\
\hline Calculate Euclidean distance & 0.1295 \\
\hline classification by using SVM Classifier & 1.1924 \\
\hline
\end{tabular}

In all the conducted experiments, the PCA and the Euclidean Distance with SVM classifier turn out to have the best result compared to the results of previous research using the same dataset. When SVM Classifier was used, we got the best recognition rate, which is $100 \%$ and the best accuracy rate which is $99.32 \%$ as shown as Table (2). Figure 8 shows an example of the matched test image.
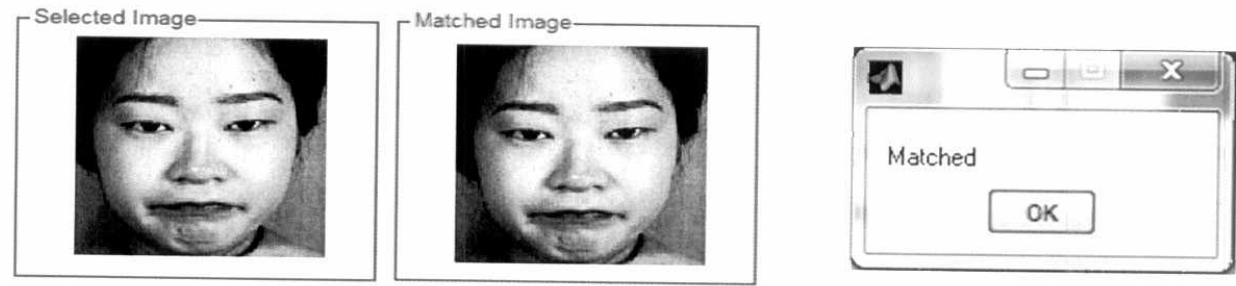

Figure 8. Result of the matched image with " expression = anger"

Accuracy is a description of systematic errors, a measure of statistical bias. The accuracy is the proportion of true results (both true positives and true negatives) among the total number of cases examined. Accuracy rate is calculated as show as:

$$
\text { Accuracy }=\frac{T P+T N}{T P+T N+F P+F N}
$$

Where TP is number of true positive, TN is number of true negative, FP is number of false positive, and FN is number of false negative. 
Table 2. Accuracy for facial expression classification

\begin{tabular}{|c|c|}
\hline SVM Classifier & Accuracy \\
\hline Neutral & $99.742 \%$ \\
\hline Anger & $99.660 \%$ \\
\hline Disgust & $98.469 \%$ \\
\hline Fear & $98.469 \%$ \\
\hline Happy & $99.688 \%$ \\
\hline Sad & $99.468 \%$ \\
\hline Surprise & $99.780 \%$ \\
\hline Average & $\mathbf{9 9 . 3 2 5 \%}$ \\
\hline
\end{tabular}

Table (3) shows a comparison with previous researches. The researches present different methods using the same database JAFFE face database with different facial expressions. Our proposed method achieved better results than previous research.

Table 3. Comparison with existing methods

\begin{tabular}{|c|c|c|}
\hline Reference & Method & Accuracy (\%) \\
\hline H.F. Huang[18] & SURF + KL divergence + WMV & $95 \%$ \\
\hline Neeta Sarode et. al.[19] & Radial Symmetry Trans & $81 \%$ \\
\hline Ying Chen[20] & $\begin{array}{c}\text { non-negative least squares } \\
\text { (NNLS) + LBP }\end{array}$ & $86.67 \%$ \\
\hline Our Method & PCA+SVM & $99.325 \%$ \\
\hline
\end{tabular}

\section{Conclusion:}

This research paper has presented a facial expression recognition system that uses Principal component analysis (PCA) that decreases the data dimensionality by performing a covariance analysis between vectors in image. The system will recognize the human facial expression for both female and male from JAFFE face dataset and, also for some face images from outside the JAFFE public dataset. We achieved good classification rate for all the facial expressions using Support Vector Machine (SVM). The system achieved superior facial expression recognition rate, Accuracy rate and reduced training time.

\section{References:}

1. S.Mehar, P.Maben.,"Face Recognition and Facial Expression Identification using PCA." IEEE International Advance Computing Conference (IACC), No. 1,pp. (1093 - 1098), 2014.

2. A.Sing, S.Kumar,"Face recognition Using PCA and Eigen Face Approach", Thesis paper, Dept. of computer Science and Engineering, National Institute of Technology, Rourkela, Vol.3, No. $769008,2012$.

3. R. Zhi, M. Flierl, Q. Ruan, and W. B. Kleijn, "Graph-Preserving Sparse Nonnegative Matrix Factorization with Application to Facial Expression Recognition", IEEE Transactions on Systems, Man and Cybernetics-Part B: Cybernetics, Vol.41, No. 1, pp. (38-52), 2011. 
4. H. Huang and H. He, "Super-Resolution Method for Face Recognition Using Nonlinear Mappings on Coherent Features",IEEE Transactions on Neural Networks, Vol. 22, No. 1, pp. (121-130), January 2011.

5. S. M. Lajevardi and H. R. Wu, "Facial Expression Recognition in Perceptual Color Space" IEEE Transactions on Image Processing, Vol. 21, No. 8, pp. (3721-3732), August 2012.

6. Abhishek S. ,Saurabh K. , "Face Recognition using PCA and Eigen Face approach",PHD Thesis,May 14 - 2012.

7. MandeepK,Rajeev V., "Comparative Study of Facial expression Recognition", International Journal of Computer Applications, Vol.13, No.1, 2011.

8. Ashraf Abbas M. Al-modwahi, et. al., "Facial Expression Recognition Intelligent security System For real Time Surveillance",Int'l Conf. Computer Graphics and Virtual Realit,Vol.12,pp.(40-47), 2012.

9. Louis A., F.O.M, EzeikielN. "Recognition of Facial Expression with PCA and Singular value Decomposition", International Journal of Statistics and Systems, Vol 9, No 2,pp. (0973-2675), 2014.

10. Seyed Mehdi L., Zahir M. H., "Local Feature Extraction method for Facial expression recognition", 17th European Signal Processing Conference (EUSIPCO 2009) Glasgow, Scotland,Vol. 17, pp. (60 - 64 ), August 24-28, 2009.

11. K.Delac ,M.Grgic, S.Grgic, " Independent Comparative Study of PCA and LDA on the FERET Dataset " University of Zagreb, FER, Vol. 15, pp.(252-260),2006.

12. B. E. Boser, I. M. Guyon, and V. N. Vapnik., "A training algorithm for optimal margin classifiers". In COLT '92: Proceedings of the fifth annual workshop on Computational learning theory, Vol.05, pp(144-152), 1992.

13. C. Hsu and C. Lin., "A comparison of methods for multi-class support vector machines",IEEE Transactions on Neural Networks, Vol.13, No.02, pp. (415-425),2001.

14. K.Crammer and Y.Singer.,"On the learn ability and design of output codes for multiclass problems“. In Computational Learning Theory, Vol.47,pp.(35-46),2002.

15. J. Platt, N. Cristianini,et. al., "Large margin DAGs for multiclass classification",In: adv. in Neural Information Processing System,Vol. 12pp. (547-533), 2000.

16. V. N. Vapnik., "Statistical Learning Theory",Wiley-Interscience,Vol.16, No.02, pp. (295- 321), September.1998.

17. J. Weston and C. Watkins. "Support vector machines for multi-class pattern recognition", European Symposium on Artificial Neural Networks (ESANN), Vol.99,pp. (219-224), 1999.

18. H.F. Huang," Facial Expression Recognition Using New Feature Extraction Algorithm", Electronic Letters on Computer Vision and Image Analysis, Vol.11, No.01, PP. (41-54); 2012.

19. Neeta Sarode et. al.,"Facial Expression Recognition" (IJCSE) International Journal on Computer Science and Engineering, Vol.02, No. 05,PP. (1552-1557), 2010.

20. Ying Chen et. al., "Facial Expression Recognition via Non-Negative Least-Squares Sparse Coding", Information, Vol.05,No.02, PP (305-318),2014. 